\title{
CHARACTERIZATIONS OF BISELECTIVE OPERATIONS
}

\author{
JIMMY DEVILLET AND GERGELY KISS
}

\begin{abstract}
Let $X$ be a nonempty set and let $i, j \in\{1,2,3,4\}$. We say that a binary operation $F: X^{2} \rightarrow X$ is $(i, j)$-selective if

$$
F\left(F\left(x_{1}, x_{2}\right), F\left(x_{3}, x_{4}\right)\right)=F\left(x_{i}, x_{j}\right),
$$

for all $x_{1}, x_{2}, x_{3}, x_{4} \in X$. In this paper we provide characterizations of the class of $(i, j)$-selective operations. We also investigate some subclasses by adding algebraic properties such as associativity or bisymmetry.
\end{abstract}

\section{INTRODUCTION}

Let $X$ be a nonempty set and let $i, j \in\{1,2,3,4\}$. We say that an operation $F: X^{2} \rightarrow X$ is $(i, j)$-selective if

$$
F\left(F\left(x_{1}, x_{2}\right), F\left(x_{3}, x_{4}\right)\right)=F\left(x_{i}, x_{j}\right),
$$

for all $x_{1}, x_{2}, x_{3}, x_{4} \in X$. Also, we say that an operation $F: X^{2} \rightarrow X$ is biselective if there exist $i, j \in\{1,2,3,4\}$ such that $F$ is $(i, j)$-selective. Among these operations, those which are $(1,3)$-selective are of particular interest as they are transitive, that is, satisfy the functional equation

$$
F(F(x, z), F(y, z))=F(x, y),
$$

for all $x, y, z \in X$ (see, e.g., [1,4] and the references therein). Also, we easily see that $(1,4)$-selective operations are bisymmetric, that is, satisfy the functional equation

$$
F(F(x, y), F(u, v))=F(F(x, u), F(y, v)),
$$

for all $x, y, u, v \in X$ (see, e.g., [1]).

In this paper we investigate the class of $(i, j)$-selective operations for every $i, j \in\{1,2,3,4\}$. In particular, we characterize these operations with and without additional properties such as associativity or bisymmetry.

The paper is organized as follows. After presenting the main definitions, we show some basic results about $(i, j)$-selective operations in Section 2. In particular, we prove that $(i, j)$-selective operations with $j<i$ are constant (see Proposition 2.4) as well as (2,3)-selective operations (see Proposotion 2.12). We also show that characterizing the $(i, j)$-selective operations is equivalent to characterizing the $(5-j, 5-i)$-selective operations (see Lemma 2.8). In Section 3 we characterize the (1,3)-selective operations (see Theorem 3.1). In Section 4 we characterize the $(1,4)$-selective operations (see Theorem 4.11) and in Section 5 we describe the $(1,2)$-selective operations in conjunction with additional properties such as

Date: June 20, 2018.

2010 Mathematics Subject Classification. Primary 39B52.

Key words and phrases. $(i, j)$-selectiveness, transitivity, axiomatization, associativity, bisymmetry. 
associativity. Finally, in Section 6 we summarize the main results and present some open questions and directions of further investigations.

\section{Preliminaries}

In this section we introduce some basic definitions and present some preliminary results.

Definition 2.1. An operation $F: X^{2} \rightarrow X$ is said to be

- idempotent if $F(x, x)=x$ for all $x \in X$,

- quasitrivial (or selective) if $F(x, y) \in\{x, y\}$ for all $x, y \in X$,

- commutative if $F(x, y)=F(y, x)$ for all $x, y \in X$,

- anticommutative if $\forall x, y \in X: F(x, y)=F(y, x) \Rightarrow x=y$,

- associative if

$$
F(x, F(y, z))=F(F(x, y), z),
$$

for all $x, y, z \in X$,

Definition 2.2. Let $F: X^{2} \rightarrow X$ be an operation.

- An element $e \in X$ is said to be a neutral element of $F$ if $F(e, x)=F(x, e)=x$ for all $x \in X$. It can be easily shown that such a neutral element is unique.

- An element $z \in X$ is said to be an annihilator of $F$ if $F(x, z)=F(z, x)=z$ for all $x \in X$. It can be easily shown that such an annihilator is unique.

- We denote the range of $F$ by $\operatorname{ran}(F)$. Clearly, $\operatorname{ran}(F)$ is nonempty since $X$ is nonempty.

- An element $x \in X$ is said to be idempotent for $F$ if $F(x, x)=x$. We denote the set of all idempotent elements of $F$ by $\operatorname{id}(F)$. Clearly, $\operatorname{id}(F) \subseteq \operatorname{ran}(F)$.

Recall that a binary relation $R$ on $X$ is said to be

- reflexive if $\forall x \in X: x R x$,

- symmetric if $\forall x, y \in X: x R y$ implies $y R x$,

- transitive if $\forall x, y, z \in X: x R y$ and $y R z$ implies $x R z$.

Recall also that an equivalence relation on $X$ is a binary relation $\sim$ on $X$ that is reflexive, symmetric, and transitive. For all $u \in X$, we use the notation $[u]_{\sim}$ to denote the equivalence class of $u$, that is, $[u]_{\sim}=\{x \in X: x \sim u\}$.

Given $F: X^{2} \rightarrow X$ we define the equivalence relation $\sim_{F}$ on $X$ by

$$
x \sim_{F} y \Leftrightarrow F(x, x)=F(y, y) \quad x, y \in X .
$$

Fact 2.3. If $F: X^{2} \rightarrow X$ is an $(i, j)$-selective operation, then $\operatorname{id}(F) \cap[x]_{\sim_{F}}=$ $\{F(x, x)\}$ for all $x \in X$.

Proposition 2.4. An operation $F: X^{2} \rightarrow X$ is an $(i, j)$-selective operation with $j<i$ if and only if $F$ is constant.

Proof. (Necessity) First, suppose that $F$ is $(4,1)$-selective (for $(3,1)-,(4,2)$ - and $(4,3)$-selective operations the proof is similar).

By Fact 2.3, we have $F(x, x) \in \operatorname{id}(F)$ for all $x \in X$. If $x, y \in \operatorname{id}(F)$, then

$$
F(x, y)=F(F(x, x), F(y, y))=F(y, x),
$$

by $(4,1)$-selectiveness. Applying this, we get

$$
x=F(x, x)=F(F(x, y), F(y, x))=F(F(y, x), F(x, y))=F(y, y)=y \text {. }
$$


Thus, $|\operatorname{id}(F)|=1$ and we can assume that $\operatorname{id}(F)=\{u\}$. Hence, by Fact 2.3 . $F(x, x)=u$ for all $x \in X$. Using $(4,1)$-selectiveness, we get

$$
F(x, y)=F(F(y, y), F(x, x))=F(u, u)=u, \quad x, y \in X .
$$

Now we suppose that $F$ is $(2,1)$-selective (the case where $F$ is $(4,3)$-selective can be dealt with similarly). By Fact 2.3 , we have $F(x, x) \in \operatorname{id}(F)$ for all $x \in X$. If $x, y \in \operatorname{id}(F)$, then

$$
x=F(x, x)=F(F(x, x), F(y, y))=F(x, y)
$$

by $(2,1)$-selectiveness. Applying that $x=F(x, y)$ and $y=F(y, x)$ for all $x, y \in$ $\operatorname{id}(F)$, we obtain

$$
x=F(x, x)=F(F(x, y), F(x, y))=F(y, x)=y .
$$

Thus, $|\operatorname{id}(F)|=1$ and we can assume that $\operatorname{id}(F)=\{u\}$. Hence, by Fact 2.3 . $F(y, y)=u$ for all $y \in X$. Using also $(2,1)$-selectiveness of $F$ we get

$$
F(y, z)=F(F(z, y), F(z, y))=u, \quad y, z \in X .
$$

(Sufficiency) Obvious.

In the following two propositions we deal with the case where $F$ is an $(i, i)$ selective operation with $i \in\{1,2,3,4\}$.

Proposition 2.5. An operation $F: X^{2} \rightarrow X$ is $(2,2)$-selective (resp. (3,3)-selective) if and only if $\left.F\right|_{\mathcal{R}^{2}(F)}$ is constant and $F(x, x) \in \operatorname{id}(F)$ for all $x \in X$.

Proof. (Necessity) Suppose that $F$ is $(2,2)$-selective (the case where $F$ is $(3,3)$ selective can be dealt with similarly). By Fact 2.3, $F(z, z) \in \operatorname{id}(F)$ for all $z \in X$. If $x, y \in \operatorname{id}(F)$, then

$$
F(x, y)=F(F(x, x), F(y, y))=F(x, x)=x .
$$

Using this and $(2,2)$-selectiveness we obtain

$$
x=F(x, x)=F(F(x, y), F(x, y))=F(y, y)=y .
$$

Thus, we can assume that $\operatorname{id}(F)=\{x\}$ and by Fact 2.3. $F(y, y)=x$ for all $y \in X$. Now let us assume that $u, v \in \operatorname{ran}(F)$. Then there exist $a, b, c, d \in X$ such that $u=F(a, b)$ and $v=F(c, d)$. Using $(2,2)$-selectiveness we obtain

$$
F(u, v)=F(F(a, b), F(c, d))=F(b, b)=x,
$$

which proves the statement.

(Sufficiency) Obvious.

Proposition 2.6. An operation $F: X^{2} \rightarrow X$ is $(1,1)$-selective (resp. (4,4)-selective) if and only if the following conditions hold.

(a) $F(x, y)=F(x, x)$ (resp. $F(x, y)=F(y, y))$ for all $x, y \in \operatorname{ran}(F)$.

(b) $F(x, y) \in[x]_{\sim_{F}}$ (resp. $F(x, y) \in[y]_{\sim_{F}}$ ) for all $x, y \in X$.

Proof. Suppose that $F$ is $(1,1)$-selective (the case where $F$ is $(4,4)$-selective can be dealt with similarly).

(Necessity) If $x, y \in \operatorname{ran}(F)$, then there exists $a, b, c, d \in X$ such that $F(x, y)=$ $F(F(a, b), F(c, d))=F(a, a)$ by $(1,1)$-selectiveness. Also, by $(1,1)$-selectiveness,

$$
F(x, x)=F(F(x, y), F(x, y))=F(F(a, a), F(a, a))=F(a, a),
$$


which gives that $F(x, y)=F(x, x)$.

Also, by $(1,1)$-selectiveness, we have $F(F(x, y), F(x, y))=F(x, x)$, which shows that $F(x, y) \in[x]_{\sim_{F}}$ for all $x, y \in X$.

(Sufficiency) Let $x, y, u, v \in X$. By condition (a), we obtain

$$
F(F(x, y), F(u, v))=F(F(x, y), F(x, y)) .
$$

Also, by condition (b), we obtain $F(F(x, y), F(x, y))=F(x, x)$.

Remark 1. In Figure1, we illustrate the partitioning of $X$ by $\sim_{F}$, where $F: X^{2} \rightarrow X$ is $(1,1)$-selective.

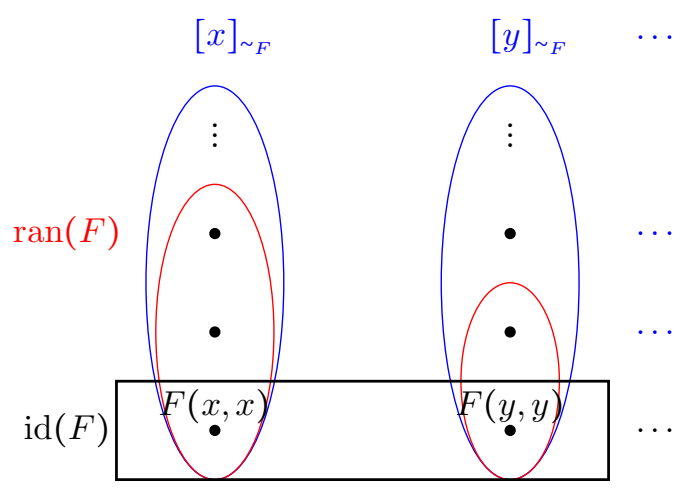

FiguRE 1.

Definition 2.7. We say that an operation $F: X^{2} \rightarrow X$ is dual transitive if

$$
F(F(x, y), F(x, z))=F(y, z),
$$

for all $x, y, z \in X$.

The following lemma shows a strong connection between $(i, j)$-selective and $(5-j, 5-i)$-selective operations. The proof is omitted as it is straightforward.

Lemma 2.8. An operation $F: X^{2} \rightarrow X$ is $(i, j)$-selective (resp. transitive) if and only if the operation $G: X^{2} \rightarrow X$ defined by $G(x, y)=F(y, x)$ for all $x, y \in X$ is $(5-j, 5-i)$-selective (resp. dual transitive).

By Lemma 2.8, all the results for $(5-j, 5-i)$-selective operations can be deduced from those for $(i, j)$-selective operations. Therefore we can focus only on $(1,2)$-, $(1,3)-,(1,4)-$, and $(2,3)$-selective operations. Now we prove some useful lemmas concerning these operations.

Recall that the projection operations are the binary operations $\pi_{1}: X^{2} \rightarrow X$ and $\pi_{2}: X^{2} \rightarrow X$ defined by $\pi_{1}(x, y)=x$ and $\pi_{2}(x, y)=y$ for all $x, y \in X$.

The following result provides a characterization of the $(1,2)$-selective operations. Its proof is omitted as it is straightforward.

Lemma 2.9. An operation $F: X^{2} \rightarrow X$ is $(1,2)$-selective if and only if $\left.F\right|_{\operatorname{ran}(F)^{2}}=$ $\left.\pi_{1}\right|_{\operatorname{ran}(F)^{2}}$. 
Lemma 2.10. Let $F: X^{2} \rightarrow X$ be an operation that is $(1,4)$-selective (resp. (1,3)selective, (2,3)-selective). For all $x, y \in X$ we have $F(x, y)=F(y, x)$ if and only if $F(x, x)=F(y, y)$. Moreover, if there exist $x, y \in X$ such that any of the previous equalities hold, then $F(x, y)=F(y, x)=F(x, x)=F(y, y)$.

Proof. We consider the case when $F$ is $(1,4)$-selective (the other cases can be dealt with similarly).

(Necessity) Suppose that $F(x, y)=F(y, x)$, then

$$
F(x, y)=F(F(x, y), F(x, y))=F(F(x, y), F(y, x))=F(x, x) .
$$

Similarly, we have that $F(x, y)=F(y, y)$.

(Sufficiency) Now assume that $F(x, x)=F(y, y)$, then

$$
F(x, x)=F(F(x, x), F(x, x))=F(F(x, x), F(y, y))=F(x, y) .
$$

Similarly, we have that $F(x, x)=F(y, x)$.

The last statement of the lemma is now immediate.

Theorem 2.11. Let $F: X^{2} \rightarrow X$ be an $(i, j)$-selective operation. Then the following assertions are equivalent.

(i) $F$ is commutative.

(ii) $|\operatorname{ran}(F)|=1$.

(iii) $F$ has an annihilator.

(iv) $|\operatorname{id}(F)|=1$.

Moreover, we have that $F$ has a neutral element if and only if $|X|=1$.

Proof. By Lemma 2.8 we only need to prove the result for $(i, j)$-selective operations where $(i, j) \in\{(1,2),(1,3),(1,4),(2,3)\}$. First, suppose that $F$ is $(1,4)$-selective (the cases where $F$ is $(1,3)$-selective or $(2,3)$-selective are similar).

(i) $\Rightarrow$ (ii). If $F$ is commutative, then by Lemma 2.10, $F(x, y)=F(x, x)$ for all $x, y \in X$. This implies that $|\operatorname{ran}(F)|=1$.

(ii) $\Rightarrow$ (iii). If $|\operatorname{ran}(F)|=1$, then $F$ has clearly an annihilator.

(iii) $\Rightarrow$ (iv). Let $a$ be the annihilator of $F$. We clearly have that $a \in \operatorname{id}(F)$. Also, if $x \in \mathrm{id}(F)$, then using $(1,4)$-selectiveness and the definition of an annihilator, we get $x=F(x, x)=F(F(x, a), F(a, x))=F(a, a)=a$ which shows that $|\operatorname{id}(F)|=1$.

(iv) $\Rightarrow$ (i). If $|\operatorname{id}(F)|=1$, then using Lemma 2.10 we get that $F$ is commutative.

Let us now prove the last part of the statement. If $|X|=1$, then $F$ has clearly a neutral element. Conversely, if $F$ has a neutral element $e \in X$, then by Lemma 2.10, $x=F(x, e)=F(e, e)=e$ for all $x \in X$ which clearly implies that $|X|=1$.

Now, suppose that $F$ is $(1,2)$-selective.

(i) $\Rightarrow$ (ii). If $a, b \in R(F)$, then using commutativity of $F$ and Lemma 2.9 we get $a=F(a, b)=F(b, a)=b$, which shows that $|\operatorname{ran}(F)|=1$.

(ii) $\Rightarrow$ (iii). If $|\operatorname{ran}(F)|=1$, then $F$ has clearly an annihilator.

(iii) $\Rightarrow$ (iv). Let $a$ be the annihilator of $F$. We clearly have that $a \in \operatorname{id}(F)$. Also, if $x \in \mathrm{id}(F)$, then using $(1,2)$-selectiveness and the definition of an annihilator, we get $x=F(x, x)=F(F(x, x), F(a, a))=F(x, a)=a$, which shows that $|\operatorname{id}(F)|=1$.

(iv) $\Rightarrow$ (i). If $\operatorname{id}(F)=\{c\}$, then using $(1,2)$-selectiveness we get

$$
F(x, y)=F(F(x, y), F(x, y))=c=F(F(y, x), F(y, x))=F(y, x),
$$

for all $x, y \in X$. 
Let us now prove the last part of the statement. If $|X|=1$, then $F$ has clearly a neutral element. Conversely, if $F$ has a neutral element $e \in X$, then for all $x \in X$ we have $F(x, e)=x \in \operatorname{ran}(F)$. On the other hand, by Lemma 2.9] $F(e, x)=e$ for all $x \in \operatorname{ran}(F)$ which implies that $|X|=1$.

As an important consequence of Theorem 2.11, we provide the characterization of $(2,3)$-selective operations.

Proposition 2.12. An operation $F: X^{2} \rightarrow X$ is $(2,3)$-selective if and only if $|\operatorname{ran}(F)|=1$.

Proof. (Necessity) We first show that $F(x, y)=F(y, x)$ for all $x, y \in X$. Using four times (2,3)-selectiveness we obtain

$$
\begin{aligned}
F(x, y) & =F(F(x, x), F(y, y)) \\
& =F(F(F(y, x), F(x, y)), F(F(x, y), F(y, x))) \\
& =F(F(x, y), F(x, y))=F(y, x),
\end{aligned}
$$

which proves the commutativity of $F$. Now, it follows from Theorem 2.11 that $|\operatorname{ran}(F)|=1$.

(Sufficiency) Obvious.

\section{3. (1,3)-SELECTIVENESS}

In the following result we provide a characterization of $(1,3)$-selective operations. In the following $\sim_{F}$ denotes the same equivalence relation as in Section 2 .

Theorem 3.1. Let $F: X^{2} \rightarrow X$ be an operation. Then, the following assertions are equivalent.

(i) $F$ is $(1,3)$-selective.

(ii) $F(x, y)=F(u, v) \in[x]_{\sim_{F}}$ for all $x, y \in X, u \in[x]_{\sim_{F}}$, and $v \in[y]_{\sim_{F}}$.

(iii) $F(F(x, y), z)=F(x, z)$ and $F(x, F(y, z))=F(x, y)$ for all $x, y, z \in X$.

Proof. (i) $\Rightarrow$ (ii). Let $x, y \in X, u \in[x]_{\sim_{F}}$ and $v \in[y]_{\sim_{F}}$. Using (1,3)-selectiveness we get

$$
F(x, y)=F(F(x, x), F(y, y))=F(F(u, u), F(v, v))=F(u, v) .
$$

Also, using (1,3)-selectiveness, we get $F(F(x, y), F(x, y))=F(x, x)$, which shows that $F(x, y)=F(u, v) \in[x]_{\sim_{F}}$.

(ii) $\Rightarrow$ (iii). Let $x, y, z \in X$. Clearly, $x \in[x]_{\sim_{F}}, z \in[z]_{\sim_{F}}$, and by (ii) we get $F(x, y) \in[x]_{\sim_{F}}$ and $F(y, z) \in[y]_{\sim_{F}}$. Thus, by (ii), we obtain $F(F(x, y), z)=F(x, z)$ and $F(x, F(y, z))=F(x, y)$.

(iii) $\Rightarrow$ (i). Let $x, y, u, v \in X$. By (iii), we obtain

$$
F(F(x, y), F(u, v))=F(F(x, y), u)=F(x, u) \text {, }
$$

which concludes the proof.

Remark 2. In Figure 2, we illustrate the partitioning of $X$ by $\sim_{F}$, where $F: X^{2} \rightarrow X$ is $(1,3)$-selective.

Corollary 3.2. If $F: X^{2} \rightarrow X$ is a $(1,3)$-selective operation, then $F(x, x)=F(y, z)$ for all $x \in X$ and $y, z \in[x]_{\sim_{F}}$. 


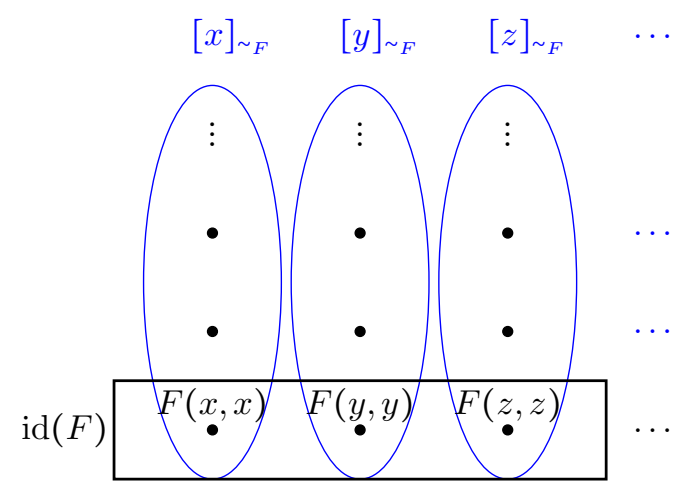

FiguRE 2.

Now if we assume that $F$ is surjective, then we easily derive the following characterization from Theorem 3.1 .

Corollary 3.3. An operation $F: X^{2} \rightarrow X$ is $(1,3)$-selective and surjective if and only if the following conditions hold.

(a) $F(x, y)=F(u, v) \in[x]_{\sim_{F}}$, for all $x, y \in X, u \in[x]_{\sim_{F}}$, and $v \in[y]_{\sim_{F}}$.

(b) For every $x \in X$ there exists $a, b \in X$ such that $F(F(a, a), F(b, b))=F(a, b)=$ $x \in[a]_{\sim_{F}}$.

Remark 3. Using condition (a) of Corollary 3.3 we can reformulate condition (b) of Corollary 3.3 in the following way. If a $(1,3)$-selective operation $F$ is surjective, then for every $x \in X$ there exists $y \in X$ such that $F(x, y)=x$.

The following result shows that associativity and bisymmetry are equivalent under $(1,3)$-selectiveness.

Proposition 3.4. Let $F: X^{2} \rightarrow X$ be an $(1,3)$-selective operation. Then the following assertions are equivalent.

(i) $F$ is associative,

(ii) $F(x, y)=F(x, z)$ for all $x, y, z \in X$,

(iii) $F$ is bisymmetric,

(iv) $\left.F\right|_{\operatorname{ran}(F) \times X}=\left.\pi_{1}\right|_{\operatorname{ran}(F) \times X}$.

Proof. (i) $\Rightarrow$ (ii). This follows from Theorem 3.1

(ii) $\Rightarrow$ (iii). Let $x, y, u, v \in X$. Using $(1,3)$-selectiveness and condition (ii), we obtain

$$
F(F(x, y), F(u, v))=F(x, u)=F(x, y)=F(F(x, u), F(y, v)),
$$

which shows that $F$ is bisymmetric.

(iii) $\Rightarrow$ (iv). Let $x, y, z \in X$. By $(1,3)$-selectiveness, $[z]_{\sim_{F}}=[F(z, y)]_{\sim_{F}}$ for all $y \in X$. Using Theorem 3.1, bisymmetry and (1,3)-selectiveness, we obtain

$$
F(F(x, y), z)=F(F(x, y), F(z, y))=F(F(x, z), F(y, y))=F(x, y) .
$$

(iv) $\Rightarrow$ (i). Let $x, y, z \in X$. By Theorem 3.1 we have $F(x, y), F(x, z) \in[x]_{\sim_{F}}$. Thus, using Theorem 3.1 and condition $(i v)$, we obtain

$$
F(F(x, y), z)=F(x, z)=F(F(x, z), F(y, z))=F(x, F(y, z)) .
$$


The following result provides characterizations of idempotent $(1,3)$-selective operations.

Proposition 3.5. Let $F: X^{2} \rightarrow X$ be an $(1,3)$-selective operation. Then the following assertions are equivalent:

(i) $F$ is quasitrivial.

(ii) $F$ is idempotent.

(iii) $\left|[x]_{\sim_{F}}\right|=1$ for all $x \in X$.

(iv) $F=\pi_{1}$.

(v) $F$ is anticommutative.

Proof. (i) $\Rightarrow$ (ii). Obvious.

(ii) $\Rightarrow$ (iii). Obvious.

(iii) $\Rightarrow$ (iv). Let $x, y \in X$ with $x \neq y$. Since $\left|[x]_{\sim_{F}}\right|=\left|[y]_{\sim_{F}}\right|=1$, we clearly have that $x$ (resp. $y$ ) is the unique element of $[x]_{\sim_{F}}$ (resp. $[y]_{\sim_{F}}$ ). Also, by Theorem 3.1 we have $F(x, y) \in[x]_{\sim_{F}}$ and $F(y, x) \in[y]_{\sim_{F}}$ and hence $F(x, y)=x$ and $F(y, x)=y$.

(iv) $\Rightarrow(\mathrm{v})$. Obvious.

$(\mathrm{v}) \Rightarrow(\mathrm{i})$. We proceed by contradiction. Suppose that there exist $x, y \in X$ such that $F(x, y) \notin\{x, y\}$. We clearly have $x \in[x]_{\sim_{F}}$ and by Theorem 3.1 we have $F(x, y) \in[x]_{\sim_{F}}$. Thus, using Corollary 3.2, we obtain $F(F(x, y), x)=F(x, x)=$ $F(x, F(x, y))$, a contradiction.

As an application of the structural description (see Theorem 3.1 and Figure 2) we can get the following results.

Proposition 3.6. Let $F$ be a $(1,3)$-selective operation on a finite $X$. Then

$$
|\operatorname{ran}(F)| \leq|\operatorname{id}(F)|^{2} \text {. }
$$

Proof. By Fact 2.3. since $F$ is $(1,3)$-selective, $F(y, y), F(z, z) \in \operatorname{id}(F)$ for all $y, z \in$ $X$. This clearly implies that the number of ordered pairs of $\operatorname{id}(F)$ cannot be smaller than $|\operatorname{ran}(F)|$.

The set $\mathrm{id}(F)$ can be listed as

$$
\operatorname{id}(F)=\left\{x_{1}, \ldots, x_{k}\right\}
$$

for some $k \geq 1$. We denote the cardinality of $\left[x_{i}\right]_{\sim_{F}}$ by $l_{i}$ for all $i \in\{1, \ldots, k\}$.

Corollary 3.7. Let $F$ be a surjective $(1,3)$-selective operation on a finite $X$ of cardinality $n \geq 1$ and $k=|\operatorname{id}(F)|$. Then $n \leq k^{2}$ and $l_{i} \leq k$ for all $1 \leq i \leq k$.

Proof. This follows from Proposition 3.6 and Corollary 3.3 .

Let $s_{k}(n)$ denote the number of $(1,3)$-selective and surjective operations on an $n$-element set $X$ with $|\operatorname{id}(F)|=k$. By Corollary 3.7, we have $s_{k}(n)=0$ if $n>k^{2}$. Finding a closed-form expression for the number of $(1,3)$-selective or surjective $(1,3)$-selective operations seems hopeless. As an illustration of the characterization given in Theorem 3.1, we calculate $s_{k}\left(k^{2}\right)$.

Proposition 3.8. For all integer $k \geq 1$, we have

$$
s_{k}\left(k^{2}\right)=\frac{k^{2} !}{((k-1) !)^{k-1}} .
$$


Proof. We have $n=k^{2}$ and $k=|\operatorname{id}(F)|$. Hence by Corollary 3.7, $l_{i}=k$ for all $1 \leq i \leq k$ (see Figure 3).

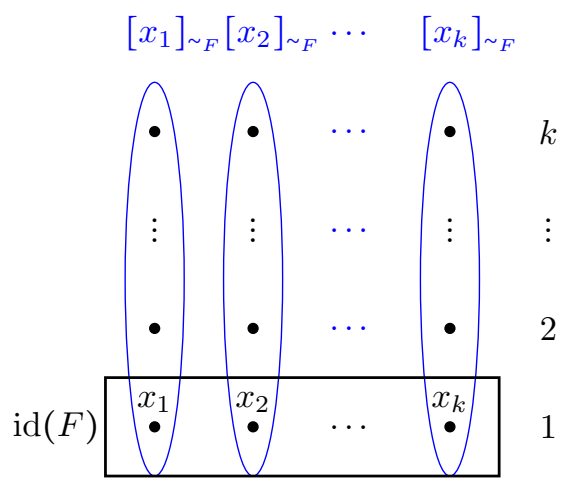

FiguRE 3.

Let $X$ be a set with $k^{2}$ elements. First we choose the equivalence classes for $\sim_{F}$. All of them have $k$ elements, thus this can be made by the multinomial coefficient $\frac{k^{2} !}{(k !)^{k}}$. Now we choose one element from each class that is also in $\operatorname{id}(F)$. This can be done in $k^{k}$ different ways.

By Theorem 3.1 and surjectivity of $F$, for all $x_{i} \in \operatorname{id}(F)$ the elements of $\left[x_{i}\right]_{\sim_{F}}$ are of the form in $F\left(x_{i}, x_{j}\right)$ for some $x_{j} \in \operatorname{id}(F)$. Since $l_{i}=|\operatorname{id}(F)|$, this implies that $F\left(x_{i}, \cdot\right)$ is a bijection between $\operatorname{id}(F)$ and $\left[x_{i}\right]_{\sim_{F}}$ with a fixed point $x_{i} \in \operatorname{id}(F)$. Thus for each $i \in\{1, \ldots, k\}$ there are $(k-1)$ ! different permutations. Consequently,

$$
s_{k}\left(k^{2}\right)=\frac{k^{2} !}{(k !)^{k}} \cdot k^{k} \cdot k(k-1) !=\frac{k^{2} ! \cdot k}{((k-1) !)^{k-1}} .
$$

Remark 4. We observe that the number of isomorphism type of $(1,3)$-selective and surjective operations on a $k^{2}$-element set $X$ with $|\operatorname{id}(F)|=k$ is 1 .

\section{4. (1,4)-SELECTIVENESS}

In this section we characterize the class of operations $F: X^{2} \rightarrow X$ that are $(1,4)$ selective (see Theorem 4.11).

Clearly, any operation $F: X^{2} \rightarrow X$ that is diagonal bisymmetric is bisymmetric. The following result provides a characterization and partial description of the class of $(1,4)$-selective operations.

Proposition 4.1. Let $F: X^{2} \rightarrow X$ be an operation. Then, the following assertions are equivalent.

(i) $F$ is $(1,4)$-selective.

(ii) $\left.F\right|_{R(F)^{2}}$ is $(1,4)$-selective and $F(x, y)=F(F(x, x), F(y, y))$ for all $x, y \in X$.

(iii) $F(F(x, y), z)=F(x, F(y, z))=F(x, z)$ for all $x, y, z \in X$.

Proof. (i) $\Rightarrow$ (ii). Obvious. 
(ii) $\Rightarrow$ (iii). Let $x, y, z \in X$ and let us prove that $F(F(x, y), z)=F(x, z)$ (the other case can be dealt with similarly). Using our assumptions, we get

$$
\begin{aligned}
F(F(x, y), z) & =F(F(F(x, y), F(x, y)), F(z, z)) \\
& =F(F(F(x, y), F(x, y)), F(F(z, z), F(z, z))) \\
& =F(F(x, y), F(z, z)) \\
& =F(F(F(x, x), F(y, y)), F(F(z, z), F(z, z))) \\
& =F(F(x, x), F(z, z))=F(x, z),
\end{aligned}
$$

which concludes the proof.

(iii) $\Rightarrow$ (i). Let $x, y, u, v \in X$. By assumption, we have $F(F(x, y), F(u, v))=$ $F(x, F(u, v))=F(x, v)$, which shows that $F$ is $(1,4)$-selective.

Corollary 4.2. If $F: X^{2} \rightarrow X$ is $(1,4)$-selective, then it is associative.

As another consequence we can prove the following.

Proposition 4.3. An operation $F: X^{2} \rightarrow X$ is $(1,4)$-selective and quasitrivial if and only if $F=\pi_{1}$ or $F=\pi_{2}$.

Proof. (Necessity) Let $x, y \in X, x \neq y$, then we have $F(x, y) \in\{x, y\}$. We can suppose without loss of generality that $F(x, y)=x$ (the other case can be dealt with similarly). Then, by Proposition 4.1 we have $F(F(x, z), y)=F(x, y)=x$ for all $z \in X$. Thus, by quasitriviality we have $F(x, z)=x$ for all $z \in X$.

(Sufficiency) Obvious.

Remark 5. We observe that quasitriviality cannot be relaxed into idempotency in Theorem 4.3. Indeed, let us consider $X=\{a, b, c, d\}$ and the operation $F: X^{2} \rightarrow X$ defined by $F(a, u)=F(c, u)=a$ and $F(b, u)=F(d, u)=d$ for all $u \in\{a, d\}$ and by $F(a, v)=F(c, v)=c$ and $F(b, v)=F(d, v)=b$ for all $v \in\{b, c\}$. It is not difficult to see that $F$ is idempotent and $(1,4)$-selective, however it is neither $\pi_{1}$ nor $\pi_{2}$. It is also not hard to show that any idempotent and $(1,4)$-selective operation on $X$, that is not quasitrivial, can be given as the previous operation after a suitable permutation of the elements $a, b, c, d$.

Now we provide a characterization of $(1,4)$-selective operations using equivalence relations.

Given $F: X^{2} \rightarrow X$ we define two binary relations $\sim_{F, 1}$ and $\sim_{F, 2}$ on $X$ by

$$
x \sim_{F, 1} y \Leftrightarrow F(x, y)=x \quad x, y \in X
$$

and

$$
x \sim_{F, 2} y \Leftrightarrow F(x, y)=y \quad x, y \in X .
$$

Thus, given $F: X^{2} \rightarrow X$ we have that $\left.F\right|_{\{x, y\}^{2}}=\left.\pi_{1}\right|_{\{x, y\}^{2}}$ (resp. $\left.F\right|_{\{x, y\}^{2}}=$ $\left.\left.\pi_{2}\right|_{\{x, y\}^{2}}\right)$ for all $x, y \in X$ with $x \sim_{F, 1} y$ (resp. $x \sim_{F, 2} y$ ).

Remark 6 . We observe that for all $F: X^{2} \rightarrow X$ the binary relation $\sim_{F, 2}$ on $X$ was already introduced in [3, Definition 2.4] and was called the trace of $F$.

Lemma 4.4. Let $F: X^{2} \rightarrow X$ be a $(1,4)$-selective operation. Then, the following assertions hold.

(a) $\operatorname{ran}(F)=\operatorname{id}(F)$.

(b) $\sim_{F, 1}$ and $\sim_{F, 2}$ are transitive binary relations on $X$. 
Proof. (a). If $x \in \operatorname{id}(F)$, then we clearly have that $x \in \operatorname{ran}(F)$. Conversely, if $x \in \operatorname{ran}(F)$, then there exist $a, b \in X$ such that $x=F(a, b)$. Thus, using $(1,4)$ selectiveness, we get $F(x, x)=F(F(a, b), F(a, b))=F(a, b)=x$, which concludes the proof.

(b). We only prove that $\sim_{F, 1}$ is transitive (the other case can be dealt with similarly). Let $x, y, z \in X$ such that $x \sim_{F, 1} y$ and $y \sim_{F, 1} z$, that is, $F(x, y)=x$ and $F(y, z)=y$. Using diagonal bisymmetry, we get

$$
F(x, z)=F(F(x, y), F(y, z))=F(x, y)=x,
$$

that is, $x \sim_{F, 1} z$.

Remark 7. We observe that Lemma 4.4(ii) is still valid if the operation $F$ is only associative.

Proposition 4.5. Let $F: X^{2} \rightarrow X$ be $(1,4)$-selective. Then, the following assertions are equivalent.

(i) $F$ is anticommutative.

(ii) $F$ is surjective.

(iii) $F$ is idempotent.

(iv) $\sim_{F, 1}$ is an equivalence relation on $X$.

(v) $\sim_{F, 2}$ is an equivalence relation on $X$.

Proof. (i) $\Rightarrow$ (ii). Suppose to the contrary that $F$ is not surjective and hence not idempotent. Thus, there exists $x \in X$ such that $F(x, x) \neq x$. Using Proposition 4.1. we obtain $F(F(x, x), x)=F(x, x)=F(x, F(x, x))$, a contradiction.

(ii) $\Rightarrow$ (iii). This follows from Lemma 4.4(a).

(iii) $\Rightarrow$ (iv). $\sim_{F, 1}$ is clearly reflexive since $F$ is idempotent. Also, by Lemma 4.4(b), we have that $\sim_{F, 1}$ is transitive. Now, let us show that $\sim_{F, 1}$ is symmetric. Let $x, y \in X$ such that $x \sim_{F, 1} y$, that is, $F(x, y)=x$. Then, using idempotency and $(1,4)$-selectiveness, we get

$$
F(y, x)=F(F(y, y), F(x, y))=F(y, y)=y,
$$

that is, $y \sim_{F, 1} x$.

(iv) $\Rightarrow$ (v). $\sim_{F, 2}$ is clearly reflexive since $\sim_{F, 1}$ is reflexive. Also, by Lemma 4.4(b) we have that $\sim_{F, 2}$ is transitive. Now, let us show that $\sim_{F, 2}$ is symmetric. Let $x, y \in X$ such that $x \sim_{F, 2} y$, that is, $F(x, y)=y$. By Proposition 4.1 we have that $y \sim_{F, 2} F(y, x)$ and by transitivity of $\sim_{F, 2}$ we get $x \sim_{F, 2} F(y, x)$, that is, $F(x, F(y, x))=F(y, x)$. By Proposition 4.1 and symmetry of $\sim_{F, 1}$, we also have that $x \sim_{F, 1} F(y, x)$, that is, $F(x, F(y, x))=x$. Hence $F(y, x)=x$, that is, $y \sim_{F, 2} x$.

$(\mathrm{v}) \Rightarrow(\mathrm{i})$. Let $x, y \in X$ such that $F(x, y)=F(y, x)$. Since $\sim_{F, 2}$ is an equivalence relation on $X$, it follows that $F$ is idempotent. Thus, using $(1,4)$-selectiveness, we obtain

$$
x=F(x, x)=F(F(x, y), F(y, x))=F(F(y, x), F(x, y))=F(y, y)=y,
$$

which concludes the proof.

The following result can be easily derived from Lemma 4.4(a) and Proposition 4.5 .

Corollary 4.6. If $F: X^{2} \rightarrow X$ is $(1,4)$-selective, then $\left.F\right|_{\operatorname{ran}(F)^{2}}$ satisfies any of the conditions $(i)-(v)$ of Proposition 4.5 . 
The following lemma is a consequence of [6, Lemma 1] and [5, Lemma 2].

Lemma 4.7. Let $F: X^{2} \rightarrow X$ be associative and idempotent. Then, the following assertions are equivalent.

(i) $F(F(x, y), z)=F(x, z)$ for all $x, y, z \in X$.

(ii) $F(F(x, y), x)=x$ for all $x, y \in X$.

(iii) $F$ is anticommutative.

Proposition 4.8. An operation $F: X^{2} \rightarrow X$ is $(1,4)$-selective and satisfies any of the conditions $(i)-(v)$ of Proposition 4.5 if and only if it is associative, idempotent, and satisfies any of the conditions $(i)-($ iii) of Lemma 4.7.

Proof. (Necessity) This follows from Corollary 4.2 and Proposition 4.5.

(Sufficiency) This follows from Lemma 4.7 and Propositions 4.1 and 4.5

Proposition 4.9. Let $F: X^{2} \rightarrow X$ be an operation. The following assertions are equivalent.

(i) $F$ is $(1,4)$-selective and satisfies any of the conditions $(i)-(v)$ of Proposition 4.5 .

(ii) $\sim_{F, 1}$ and $\sim_{F, 2}$ are equivalence relations on $X$ such that $[x]_{\sim_{F, 2}} \cap[y]_{\sim_{F, 1}}=$ $\{F(x, y)\}$ for all $x, y \in X$.

(iii) The following conditions hold.

(a) $\sim_{F, 1}$ and $\sim_{F, 2}$ are equivalence relations on $X$.

(b) For all $x, y, z \in X$ such that $x \in[y]_{\sim_{F, 1}}$ there exists a unique $u \in[z]_{\sim_{F, 1}}$ such that $x \sim_{F, 2} u$.

(c) $F(x, y)=F(x, z)$ for all $x, y, z \in X$ such that $y \sim_{F, 1} z$.

Proof. (i) $\Rightarrow$ (ii): By Proposition 4.5 we have that $\sim_{F, 1}$ and $\sim_{F, 2}$ are equivalence relations on $X$. Let $x, y \in X$ and let us show that $[x]_{\sim_{F, 2}} \cap[y]_{\sim_{F, 1}}=\{F(x, y)\}$. By Proposition 4.1 we have $F(x, y) \in[x]_{\sim_{F, 2}} \cap[y]_{\sim_{F, 1}}$. Also, if $z \in[x]_{\sim_{F, 2}} \cap[y]_{\sim_{F, 1}}$, then $z \sim_{F, 1} \cap \sim_{F, 2} F(x, y)$ which by definition implies that $z=F(x, y)$.

(ii) $\Rightarrow$ (iii): Condition (a) is clearly satisfied.

Let $x, y, z \in X$ such that $x \in[y]_{\sim_{F, 1}}$. By (ii), we have $[x]_{\sim_{F, 2}} \cap[z]_{\sim_{F, 1}}=\{F(x, z)\}$, which proves condition (b).

Let $x, y, z \in X$ such that $y \sim_{F, 1} z$, that is, $[y]_{\sim_{F, 1}}=[z]_{\sim_{F, 1}}$. By (ii) and the previous assumption, we get

$$
\{F(x, y)\}=[x]_{\sim_{F, 2}} \cap[y]_{\sim_{F, 1}}=[x]_{\sim_{F, 2}} \cap[z]_{\sim_{F, 1}}=\{F(x, z)\},
$$

which proves condition (c).

(iii) $\Rightarrow$ (i): Since $\sim_{F, 1}$ and $\sim_{F, 2}$ are equivalence relations on $X$, it follows that $F$ is idempotent. Let $x, y, u, v \in X$ and let us show that $F(F(x, y), F(u, v))=F(x, v)$. We clearly have that $t \in[t]_{\sim_{F, 1}}$ for all $t \in X$. By conditions (b) and (c), we have that there exists a unique $s \in[y]_{\sim_{F, 1}}$ such that $F(x, y)=F(x, s)=s$, that is, $x \sim_{F, 2} s$. Also, by conditions (b) and (c), we have that there exists a unique $t \in[v]_{\sim_{F, 1}}$ such that $F(u, v)=F(u, t)=t$, that is, $u \sim_{F, 2} t$. Moreover, by conditions (b) and (c), we have that there exists a unique $z \in[v]_{\sim_{F, 1}}$ such that $F(s, t)=F(s, z)=z$, that is, $s \sim_{F, 2} z$. By transitivity of $\sim_{F, 2}$ we have that $x \sim_{F, 2} z$ and by condition (b) we have that $z$ is unique. Thus, we obtain $F(F(x, y), F(u, v))=F(x, v)=F(x, z)=z$ which concludes the proof.

Remark 8. In Proposition 4.9(iii), conditions (b) and (c) can be replaced by the following two conditions. 
(b') For all $x, y, z \in X$ such that $x \in[y]_{\sim_{F, 2}}$ there exists a unique $u \in[z]_{\sim_{F, 2}}$ such that $x \sim F, 1$.

(c') $F(y, x)=F(z, x)$ for all $x, y, z \in X$ such that $y \sim_{F, 2} z$.

The following corollary is an equivalent form of Proposition 4.9.

Corollary 4.10. An operation $F: X^{2} \rightarrow X$ is $(1,4)$-selective and satisfies any of the conditions $(i)-(v)$ of Proposition 4.5 if and only if the following conditions hold.

(i) $\sim_{F, 1}$ and $\sim_{F, 2}$ are equivalence relations on $X$ and for all $x, y \in X$ there exists a bijection $f:[x]_{\sim_{F, 1}} \rightarrow[y]_{\sim_{F, 1}}$ defined by

$$
f(u)=v \Leftrightarrow u \sim_{F, 2} \quad v, \quad u \in[x]_{\sim_{F, 1}}, v \in[y]_{\sim_{F, 1}} .
$$

(ii) $F(x, y)=F(x, z)$ for all $x, y, z \in X$ such that $y \sim_{F, 1} z$.

According to Corollary 4.10, any $(1,4)$-selective and idempotent operation $F: X^{2} \rightarrow$ $X$ can be represented in a grid form as follows. Two elements $x, y \in X$ belong to the same column (resp. row) if and only if $x \sim_{F, 1} y$ (resp. $x \sim_{F, 2} y$ ). Conversely, any operation $F: X^{2} \rightarrow X$ such that $\sim_{F, 1}$ and $\sim_{F, 2}$ are equivalence relations and that can be represented in such a grid form with the convention that $F(x, y)=F(x, z)$ for all $x, y, z \in X$ such that $y \sim_{F, 1} z$, is $(1,4)$-selective and idempotent (see Figure (4).

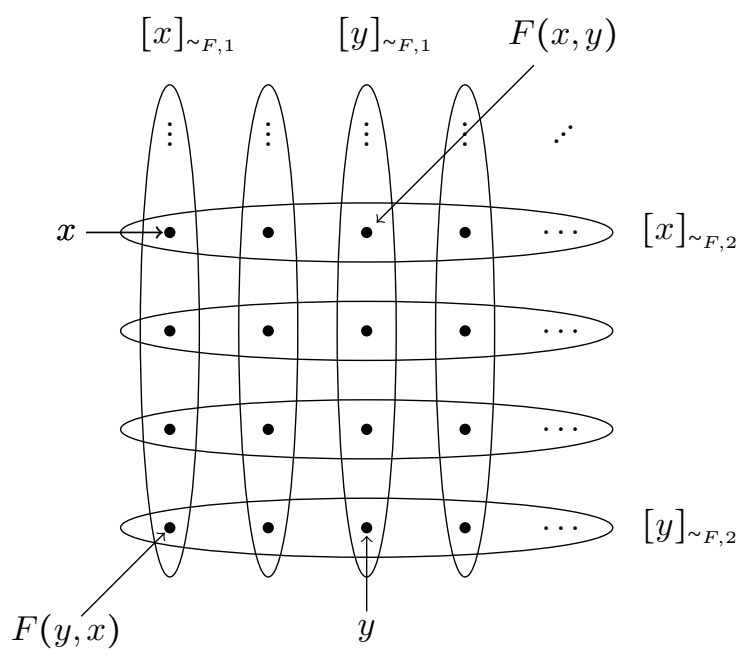

FigURE 4.

The following result, which is an immediate consequence of Propositions 4.1 . 4.5, 4.9 and Corollaries 4.6 and 4.10, provides a characterization of $(1,4)$-selective operations. 
Theorem 4.11. Let $F: X^{2} \rightarrow X$ be an operation and let $F^{\prime}=\left.F\right|_{\operatorname{ran}(F)^{2}}$. Then, the following assertions are equivalent.

(i) $F$ is $(1,4)$-selective

(ii) $F^{\prime}$ satisfies the conditions $(i)-($ iii $)$ of Proposition 4.9 and $F(u, v)=$ $F(F(u, u), F(v, v))$ for all $u, v \in X$.

(iii) $F^{\prime}$ satisfies the conditions $(i)$ and $($ ii $)$ of Corollary 4.10 and $F(u, v)=$ $F(F(u, u), F(v, v))$ for all $u, v \in X$.

For all integer $n \geq 1$, let $X_{n}=\{1, \ldots, n\}$ and let $\alpha(n)$ (resp. $\left.\beta(n)\right)$ denote the number of $(1,4)$-selective operations on $X_{n}$ that are idempotent (resp. the number of isomorphism types of $(1,4)$-selective operations on $X_{n}$ that are idempotent). In the following propositions we show that $\alpha(n)=A 121860(n)$ and $\beta(n)=d(n)=$ $A 000005(n)$ (see [7]), where $d(n)$ denotes the number of positive integer divisors of $n \in \mathbb{N}$.

Proposition 4.12. For all integer $n \geq 1$, we have

$$
\alpha(n)=\sum_{d \mid n} \frac{n !}{d !\left(\frac{n}{d}\right) !}
$$

Proof. By Corollary 4.10, counting the number of $(1,4)$-selective operations on $X_{n}$ that are idempotent is equivalent to counting the number of ways to partition $X_{n}$ into $k$ equivalence classes of sizes $l, \ldots, l$ and the number of bijections between two consecutive equivalence classes. Thus, we have

$$
\alpha(n)=\sum_{\substack{k, l \\
k l=n}} \frac{\left(\begin{array}{c}
n \\
l, \ldots, l
\end{array}\right)}{k !}(l !)^{k-1}=\sum_{\substack{k, l \\
k l=n}} \frac{n !}{k ! l !},
$$

where the multinomial coefficient $\left(\begin{array}{c}n \\ l, \ldots l\end{array}\right)$ provides the number of ways to put the elements $1, \ldots, n$ into $k$ classes of sizes $l, \ldots, l$ and $l$ ! is the number of bijections between two such classes.

Proposition 4.13. For all integer $n \geq 1$, we have $\beta(n)=d(n)$.

Proof. By Corollary 4.10, counting the number of isomorphism types of $(1,4)$ selective operations on $X_{n}$ that are idempotent is equivalent to counting the number of ways to arrange the elements of $X_{n}$ in an unlabeled grid form, where two elements $x, y \in X_{n}$ belong to the same column (resp. row) if and only if $x \sim_{F, 1} y$ (resp. $x \sim_{F, 2} y$ ). Thus, $\beta(n)$ provides the number of ways to write $n$ into a product of two elements $k, l \in\{1, \ldots, n\}$. This is in turn the number of divisors of $n$.

Corollary 4.14. $\alpha(n)=2$ (resp. $\beta(n)=2)$ if and only if $n$ is prime.

Corollary 4.15. Let $F: X_{n}^{2} \rightarrow X_{n}$ be $(1,4)$-selective and idempotent. If $n$ is prime, then $F=\pi_{1}$ or $F=\pi_{2}$.

Remark 9. From Corollary 4.15] it follows that the example of $(1,4)$-selective and idempotent operation described in Remark 5 is the smallest example that is neither $\pi_{1}$ nor $\pi_{2}$. 


\section{5. (1,2)-Selectiveness}

In Lemma 2.9 we already gave a characterization of $(1,2)$-selective operations. As a corollary we get the following result if $F$ is surjective.

Corollary 5.1. Let $F: X^{2} \rightarrow X$ be an operation that is $(1,2)$-selective. Then the following assertions are equivalent.

(i) $F=\pi_{1}$,

(ii) $F$ is quasitrivial,

(iii) $F$ is idempotent,

(iv) $F$ is surjective.

Proof. (i) $\Rightarrow$ (ii) $\Rightarrow$ (iii) $\Rightarrow$ (iv): Obvious.

(iv) $\Rightarrow$ (i): This follows from Lemma 2.9 ,

Now we characterize those operations that are bisymmetric.

Proposition 5.2. A bisymmetric operation $F: X^{2} \rightarrow X$ is $(1,2)$-selective if and only if the following two conditions hold.

(i) $F(x, y)=F(x, z)$ for all $x, y, z \in X$,

(ii) $\left.F\right|_{\operatorname{ran}(F) \times X}=\left.\pi_{1}\right|_{\operatorname{ran}(F) \times X}$.

Proof. (Necessity) Let $x, y, z \in X$. Using bisymmetry and $(1,2)$-selectiveness, we get

$$
F(x, y)=F(F(x, y), F(z, z))=F(F(x, z), F(y, z))=F(x, z),
$$

which proves (i).

Let $x \in \operatorname{ran}(F)$ and $y \in X$. Since $x \in \operatorname{ran}(F)$, there exist $x_{1}, x_{2} \in X$ such that $F\left(x_{1}, x_{2}\right)=x$. Hence, using (i) and $(1,2)$-selectiveness, we get

$$
F(x, y)=F(x, x)=F\left(F\left(x_{1}, x_{2}\right), F\left(x_{1}, x_{2}\right)\right)=F\left(x_{1}, x_{2}\right)=x,
$$

which proves (ii).

(Sufficiency) Condition (ii) clearly implies that $F$ is $(1,2)$-selective.

Let $x, y, u, v \in X$. Using condition (i) and $(1,2)$-selectiveness, we get

$$
F(F(x, y), F(u, v))=F(x, y)=F(x, u)=F(F(x, u), F(y, v)) \text {, }
$$

which shows that $F$ is bisymmetric.

In Figure 5 we illustrate a $(1,2)$-selective and bisymmetric operation on $X$. The vertical lines express that $F(x, \cdot)=x$ for all $x \in \operatorname{ran}(F)$. The dotted lines express that the function $F$ is constant along those lines.

In the following statement we provide a characterization of those operations that are associative.

Proposition 5.3. An associative operation $F: X^{2} \rightarrow X$ is $(1,2)$-selective if and only if the following two conditions hold.

(i) $F(x, y)=F(x, F(y, z))$ for all $x, y, z \in X$,

(ii) $\left.F\right|_{\operatorname{ran}(F) \times X}=\left.\pi_{1}\right|_{\operatorname{ran}(F) \times X}$.

Proof. (Necessity) Let $x \in \operatorname{ran}(F)$ and $y \in X$. By Lemma 2.9 we have $F(x, x)=x$. Thus, using associativity and $(1,2)$-selectiveness, we get

$$
\begin{aligned}
F(x, y) & =F(F(x, x), y) \\
& =F(F(F(x, x), x), y)=F(F(x, x), F(x, y))=F(x, x)=x,
\end{aligned}
$$




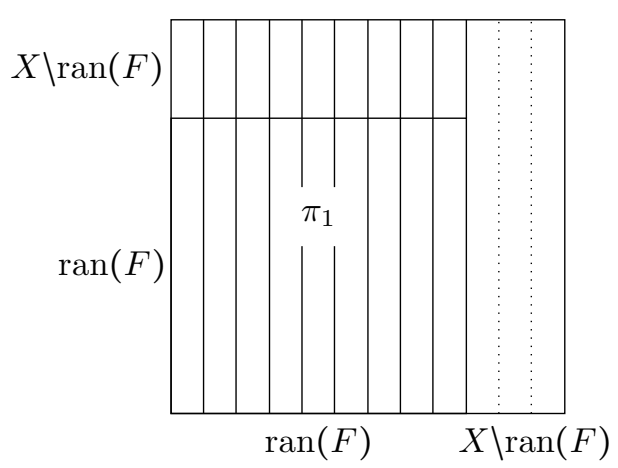

FiguRE 5 .

which proves (ii).

Let $x, y, z \in X$. Since $F(x, y) \in \operatorname{ran}(F)$, using (ii) and the associativity of $F$, we get $F(x, y)=F(F(x, y), z)=F(x, F(y, z))$, which proves (i).

(Sufficiency) Condition (ii) clearly implies that $F$ is $(1,2)$-selective.

Let $x, y, z \in X$. Applying (ii) for $F(x, y) \in \operatorname{ran}(F)$ and (i) we get $F(F(x, y), z)=$ $F(x, y)=F(x, F(y, z))$, which shows that $F$ is associative.

As a consequence of Propositions 5.2 and 5.3 we get the following.

Corollary 5.4. Any (1,2)-selective and bisymmetric operation is associative.

Finally we show an example of $(1,2)$-selective and associative operation that is not bisymmetric on $X=\{a, b, c, d\}$ (see Figure 6). The value $F(x, y)$ is represented above the corresponding point $(x, y)$ in Figure 6 for all $x, y \in X$. That is $\left.F\right|_{\mathcal{R}_{F}^{2}}=$ $\left.\pi_{1}\right|_{\mathcal{R}_{F}^{2}}$ and $F(d, a)=F(d, b)=F(d, d)=a$ and $F(d, c)=b$. By Lemma 2.9, $F$ is $(1,2)$-selective. It is also clear that $F$ is not bisymmetric by Proposition 5.2 , Using Proposition 5.2 it can be easily shown that $F$ is associative.

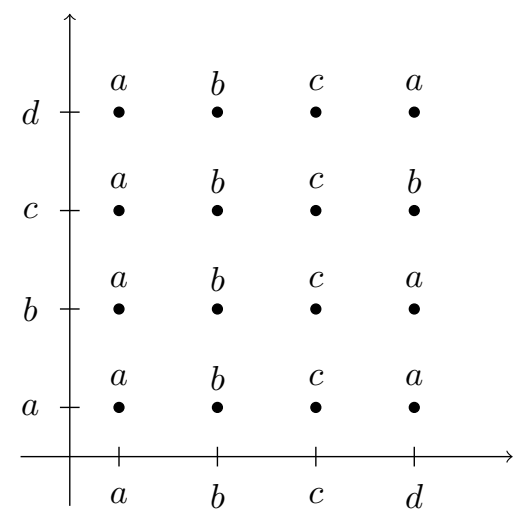

FIGURE 6. 


\section{CONCLUSiOn AND FURTher DiRECTIONS}

In this article we introduced and investigated the $(i, j)$-selective operations. First we showed some basic properties of these operations. As a consequence we proved that any $(i, j)$-selective operation with $j<i$ and any $(2,3)$-selective operation is constant. We also characterized $(i, i)$-selective operations. We described $(1,3)$ selective operations using the equivalence relation $\sim_{F}$ and it turned out that it is enough to understand $\operatorname{id}(F)$ (the set of idempotent elements). We also proved that $(1,4)$-selective operations are bisymmetric and associative. We characterized (1,4)-selective operations using equivalence relations $\sim_{F, 1}$ and $\sim_{F, 2}$. Finally we described $(1,2)$-selective operations. We studied the relation of these operations with associativity, bisymmetry and other basic properties.

In view of these results some questions arise. Now, we list them below.

- Let $n \geq 3$ be an integer and let $i_{1}, \ldots, i_{n} \in\left\{1, \ldots, n^{2}\right\}$. We say that an operation $F: X^{n} \rightarrow X$ is $\left(i_{1}, \ldots, i_{n}\right)$-selective, if

$$
F\left(F\left(x_{1}, \ldots, x_{n}\right), \ldots, F\left(x_{n(n-1)+1}, \ldots, x_{n^{2}}\right)\right)=F\left(x_{i_{1}}, \ldots, x_{i_{n}}\right),
$$

for all $x_{1}, \ldots, x_{n^{2}} \in X$.

Find characterizations of the class of $\left(i_{1}, \ldots, i_{n}\right)$-selective operations.

- Let $i, j \in\{1,2,3,4\}$. We say that the operations $F, G, H, K: X^{2} \rightarrow X$ are generalized $(i, j)$-selective if

$$
F\left(G\left(x_{1}, x_{2}\right), H\left(x_{3}, x_{4}\right)\right)=K\left(x_{i}, x_{j}\right), \quad x_{1}, x_{2}, x_{3}, x_{4} \in X .
$$

Find characterizations of the class of generalized $(i, j)$-selective operations.

- Recall that an operation $F: X^{2} \rightarrow X$ is said to be permutable [1,2, if it satisfies the following functional equation

$$
F(F(x, y), z)=F(F(x, z), y), \quad x, y, z \in X .
$$

We observe that any $(1,2)$-selective operation that is bisymmetric is permutable. Find the conditions under which an $(i, j)$-selective operation is permutable.

\section{ACKNOWLEDGEMENTS}

The authors would like to thank Jean-Luc Marichal for fruitful discussions and valuable remarks. This research is supported by the internal research project RAGR-0500 of the University of Luxembourg and by the Luxembourg National Research Fund R-AGR-3080.

\section{REFERENCES}

[1] J. Aczél. Lectures in Functional Equations and Their Applications, Dover Publications , Inc., New York, 2006.

[2] H. Bustince, M. J. Campión, F. J. Fernández, E. Induráin, and M. D.Ugarte. New trends on the permutability equation, Aequat. Math. 88 (2014), p. 211-232.

[3] J. C. Candeal and E. Induráin. Bivariate functional equations around associativity, Aequat. Math. 84 (2012), p. 137-155.

[4] Pl. Kannappan, Functional Equations and Inequalities with Applications, Springer, Inc., New York, 2009.

[5] N. Kimura. The structure of idempotent semigroups. I. Pacific J. Math., 8:257-275, 1958.

[6] D. McLean. Idempotent semigroups. Amer. Math. Monthly, 61:110-113, 1954.

[7] N.J.A. Sloane (editor). The On-Line Encyclopedia of Integer Sequences. http://www.oeis.org 
Mathematics Research Unit, University of Luxembourg, Maison du Nombre, 6, avenue De la Fonte, L-4364 Esch-Sur-Alzette, Luxembourg

E-mail address: jimmy.devillet [at]uni.lu

Mathematics Research Unit, University of Luxembourg, Maison du Nombre, 6, avenue DE LA Fonte, L-4364 Esch-SuR-Alzette, Luxembourg

E-mail address: gergely.kiss [at] uni.lu 